\title{
Intrusion Detection Prevention Systems and Performance Analysis via Internet Protocol Television (IPTV) using Snort Rule
}

\author{
Narit Hnoohom \\ Department of Computer Engineering Faculty of Engineering Mahidol University \\ Thailand \\ narit.hno@mahidol.ac.th \\ Mahasak Ketcham \\ Department of Information Technology Management Faculty of Information Technology \\ King Mongkut's University of Technology North Bangkok \\ Thailand \\ mahasak.k@it.kmutnb.ac.th
}

\begin{abstract}
This paper proposed the use of the Intrusion Prevention Systems (IPS) technique to prohibit network intrusion as well as test its performance in internal attacks. The performance analysis of the network intrusion prevention system via Internet Protocol Television (IPTV) focused on the prevention of Denial of Service (DoS) attacks, which aim to destroy the host computer that is available to users. Snort was also applied for use in IPTV. From the experimental results, the IPS of TCP protocol attacked over a wired network by using Multicast IP Address displayed the most effective results by detecting up to 39,389 attack packets. Therefore, the IPS can be applied effectively to reduce, delay and prevent network intrusions.
\end{abstract}

Keywords: Internet Protocol Television, Intrusion Prevention System, Denial of Service, TCP protocol

Received: 16 May 2018, Revised 2 July 2018, Accepted 10 July 2018

DOI: $10.6025 /$ isej/2018/5/2/49-62

(C) 2018 DLINE. All Rights Reserved

\section{Introduction}

From the resolution by the ASEAN Ministers Responsible for Information (AMRI) No. 11 on March 1, 2012 in Kuala Lumpur, Malaysia [1], it was agreed that ASEAN countries could define the end date for analogue terrestrial television during the period between 2015-2020, with subsequent modification of systems from analog to digital television standard DVB-T2. Consequently, the National Broadcasting and Telecommunication Commission (NBTC) set a plan to cease analogue terrestrial television sometime between 2012 and 2016, as required by law to proceed. It also enabled the increase of terrestrial television channels in Thailand for better quality from 6 channels to approximately 100 channels. 
The investment by manufacturers (Content Providers) in changing the system to digital television required an investment of at least 100 - 200 million baht per person for switching equipment production to digital systems. As a result, small and medium-sized entrepreneurs searched for a new kind of television broadcasting that could be watched over the internet, called internet protocol television (IPTV). In the 21st Century, the Internet has changed human civilization. Traditional television has been affected as well. Currently, numerous traditional television systems originating from cable and station broadcast have been replaced by the Internet due to the greater ease of access to new forms of technology when compared to the limitations of traditional technologies, most significantly geographic problems. This fact has lead to a new channel for watching television.

Internet protocol television covers both live broadcasts and video on demand. The main component for using IPTV is a personal computer or set-top-box connected to a television. The data requirement for signal transfer has been compressed using encoded MPEG-2 or MPEG-4, after which the compressed data is sent to MPEG Transport Stream by IP Multicast.

In the case of data transfer for a live broadcast or using IP Unicast for video, the live broadcast uses the IGMP protocol to connect to Multicast Stream and switch to other Multicast Streams. Video broadcasting uses RTSP (Real Time Streaming Protocol). From the results of a survey conducted at Point Topic Ltd. on 5 May 2016, the global IPTV market report for the period from 2014 to 2015 showed that the number of IPTV users increased 6 million in 24 months from Q3 2014 to Q4 2015, as shown in Figure 1 [2].

\section{Global IPTV Trends}

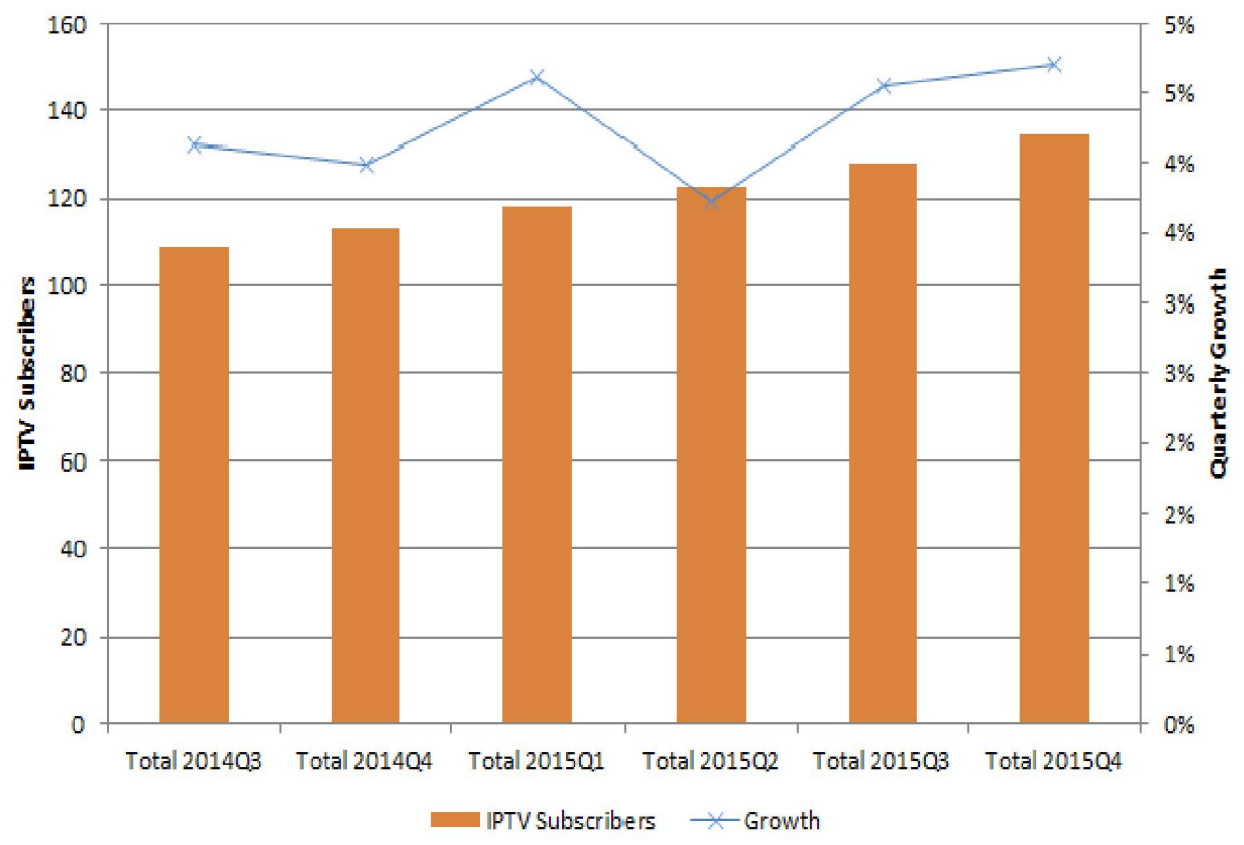

Figure 1. The number of global IPTV users in 2014-2015

However, there is still no standard for network security to confirm that IPTV is secure and effective. In attempts to remedy this oversight, viewer identification and authentication for IPTV using an RFID technique has been proposed by [3] several researchers, where an RFID system is joined with IPTV STB. Utilizing a wireless RFID tag, an authorized subscriber can be recognized and verified. Each subscriber's viewing privileges and terms of service, which enable them to view the appropriate amount of content based on agreed terms of service, are recorded on the RFID tag. Other authors [4] have suggested secure user authentication. In such a scheme, effective security and reliable authentication are guaranteed through the use of smart cards in IPTV or smart cards encoded with bio data. Based on their specific features, subscribers are given the corresponding level of content. A protected mutual validation for IPTV broadcasting has been recommended by another author [5], which applies a key exchange system between the STB and smart card to enable IPTV broadcasting. During a registration procedure, the system transmits a specific array of attributes to validate users, which are created without disclosing the identity of the user. The assertion for use of this system is that it offers efficient protection against nearly all known IPTV risks. From previous research 
[6], security of the IPTV system is important. If the system is attacked, it results in significant adverse effects on the value chain of business. Hence, IPTV systems require security technology to prevent intrusion of the network infrastructure. The copyright protection system [7] prevents the broadcasting of content due to piracy as well as unauthorized data transfers with a one-time password to log into the system [8]. Even the use of Kerberos [9], a protocol used to verify accuracy between a client and server, is not enough for prevention and verification because the current patterns of attack and terrorism on the internet have different severity levels. Some attacks may steal information, but do not affect the overall system. On the other hand, a Denial of Service (DoS) aims to destroy the open network for users by simulating it and then accessing the system simultaneously. As a consequence, the server is unable to support the use of the service or provide services as intended.

From the above issues, the intrusion prevention system (IPS) has been developed with a firewall to examine the characteristics of users' information. The system has filtering accuracy. If the characteristics of a user's information are unusual, the IPS will delete the information immediately. The analysis of IPS position in IPTV is proposed for a comparison of performance by the IPS as the most effective in the IPTV network infrastructure.

\section{Related Work}

Tibin [6] proposed an analysis of technology and security requirements in a business environment. His study summarized the performance of the security requirements for IPTV. In that security analysis, the IPTV component had some defects. Security components are essential for the protection of individual components and can affect the value creation chain in the business. Cho D-E. and Yeo S. S [7] proposed the D - CAS and DRM system, which provide security for downloading content. Many digital data sources contain copyrights to limit the content available to users with the correct permissions. It also protects data for security as technology of the copyrighted content. Chung, M. et al. [8] proposed a one-time password (OTP) to help improve security and reduce the risk of content theft by entering an OTP. In its performance and security, it limits users' requirements for breaking into the network of IPTV. Doh, I. et al. [9] proposed using Kerberos, which is a protocol that verifies accuracy between a client and server via an entrusted third party with access to the open network environment. The third party provides the secret key to all clients and verifies its authenticity. Pomsathit, A [10] proposed the performance of the IDS with a honeypot in networking. The goal was to detect the IDS more efficiently in comparison with implementation and IDS with a honeypot. The system operated under the Linux operating system, consisting of two primary parts; the IDS and database system.

From related research, security and network-based intrusion prevention systems (NIPS) contain major issues of focus for entrepreneurs because there is a lack of services provision as well as a negative effect on the reliability of the business. As a result, researchers developed security researches for applying various technologies into the application. At present, NIPS has been developed concurrently with new technology. For example, IPTV has been developed with service security. From related research, it is apparent that there is no security in IPTV that is sufficient for entrepreneurs. Nonetheless, the cost of investing in network security systems is high. Thus, NIPS applications using Open Source Snort installed on the Linux Ubuntu operating system with Snort Rule Base has been used to monitor users' service packages.

\section{Proposed Method}

In operation, NIPS is installed in the network infrastructure of IPTV. Intrusion performance is tested on an internal attack of the network via the network system and Denial of Service (Dos) with ICMP, TCP and UDP protocol. It analyzes the attack prevention involving Unicast Attack and Multicast Attack. The method is classified as follows:

\subsection{Design and Development of the Network Intrusion Prevention System Process}

The development of NIPS involves identifying intruders who delay, suspend or interfere with the actions of the server computer system for IPTV.

The design of the network infrastructure of IPS for IPTV is used to test and analyze the effectiveness of the sensors used in the IDS and IPS from malicious individuals from both inside and outside the organization. It is also designed in a laboratory to be close to an actual system in order to be developed for practical use.

Figure 2 shows the network infrastructure of IPTV, which is a simulation system for its services. It consists of two parts: an internet data center and internal network. The internet data center serves as the broadcast to users, while the internal network serves as a content production to further send to internet data center. 


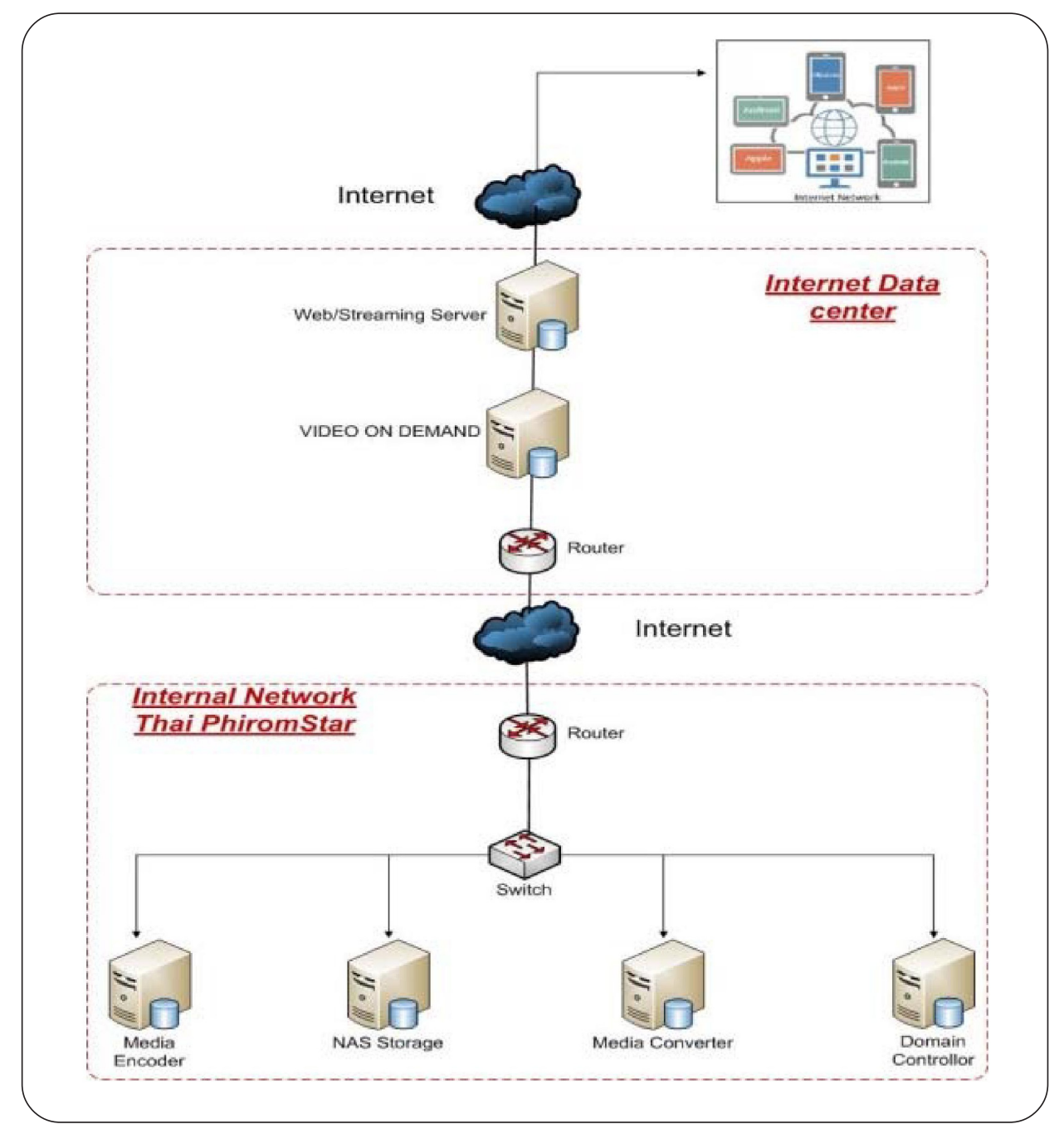

Figure 2. Network infrastructure for IPTV

\subsection{The work program for the IPS}

From previous research [11], researchers designed the work program for the IPS with the goal of detecting network intrusions more efficiently and without only using the Snort Sensor, which has components as shown in Figure 3. There are two main parts, consisting of IPS and the database, as described below.

\subsubsection{IPS}

Snort runs on the Linux operation system, which is an open source. It has an important file, snort.conf, used to configure to work with the research and snort rule, which can be edited.

\subsubsection{Database}

The database is a structured set of MySQL data that can support the Linux operation system and works with snort as well. PhpMyAdmin program helps to manage the snort's database and displays the output to a web page. In order to show the output, it needs to have a web server. Thus, Apache is used for installing the web server. The display of the web page is written by PHP and can bring the data from the web server to the web page.

Figure 3 shows the structural design for the work program of the IPS. Snort runs on free software called the Linux Fedora operation system. There are two parts that are installed, including the IPS sensor, which is a part of snort, and the database, which stores the snort rule base. 


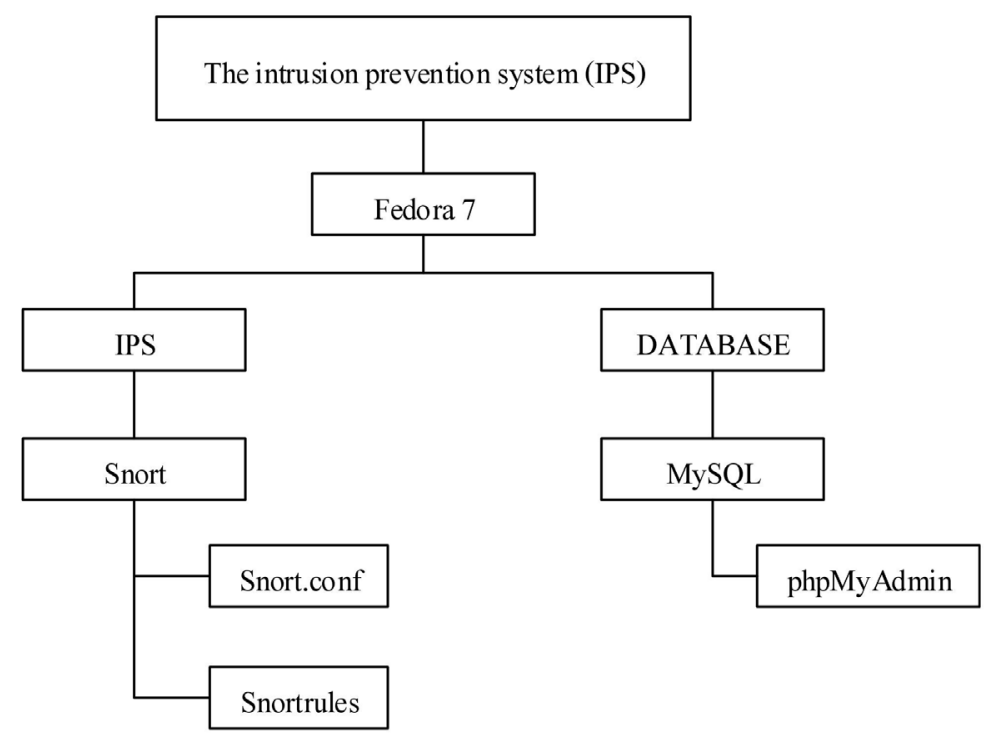

Figure 3. Structural design for the IPS work program

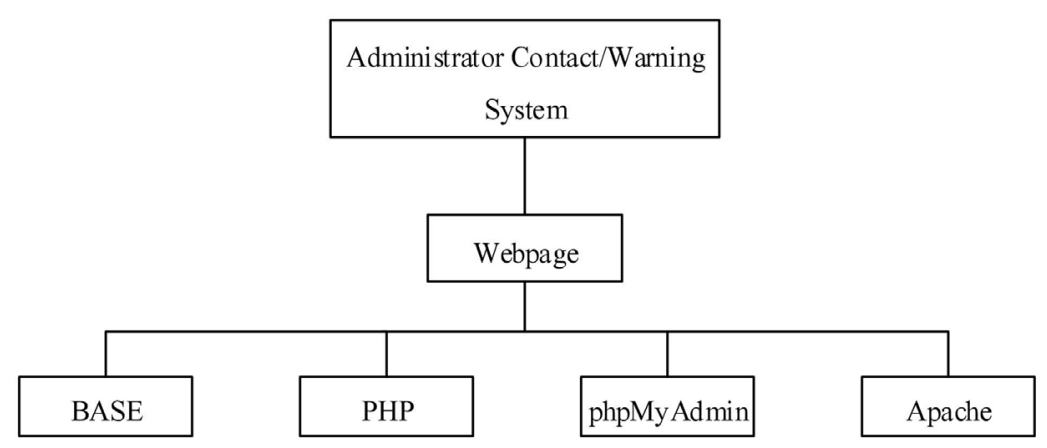

Figure 4. Structural design for the IPS work program

\subsection{Methodology}

The main function of the IDS is to detect network intrusion more efficiently by analyzing the attack through the network and wireless network protocol including ICMP, TCP and UDP. Unicast and Multicast are used for analyzing the attack prevention.

\subsubsection{Program and Software Installation}

3.3.1.1 Linux Operation System

Linux - Ubuntu family of operating systems is installed for testing.

\subsubsection{Snort Installation}

Snort is network security software that has been developed globally to its present Version 2.8.0.1, meaning that it has established itself as standardized software. It runs on the Linux operating system.

\subsubsection{Snort Database in MySQL}

Snort can store data in any available database with a variety of software. An open source often chosen for handling databases is MySQL because it can be developed with source code. Another program is PhpMyAdmin, which also allows easier management of a database.

\subsubsection{Snort Rule Installation}

The rule is a regulation to detect attack patterns or packet detection. The snort rule has been configured and customized 
because the rule from the program analyzes only simple attack forms. The program will enable operation during some forms of intensified attack. However, it cannot compare the highest performance in detecting an attack on the program. Thus, a new rule is set by enabling all programs that can be detected, while modifying and configuring the Snort.conf file.

\subsubsection{Network System Design}

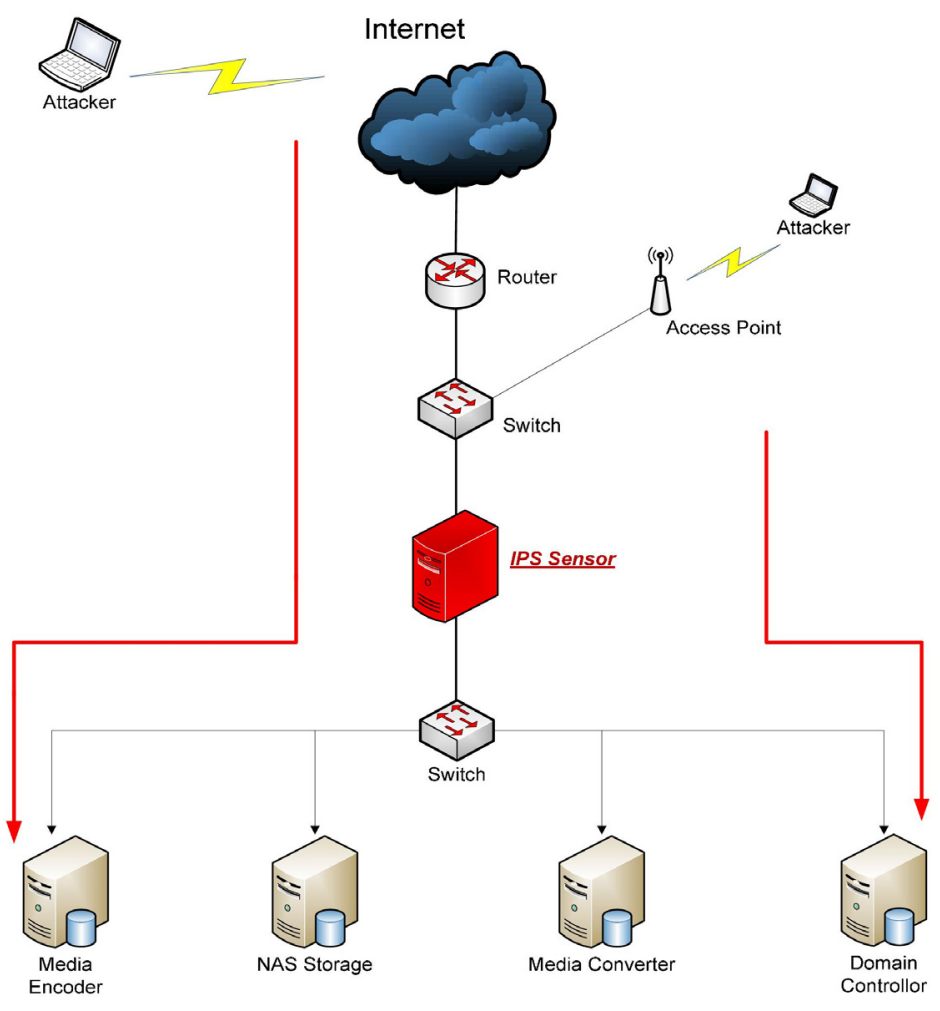

Figure 5. Network system design for IPS Sensor

Figure 5 shows the network system design. The IPS Sensor is designed in the internal network and installed in front of the switch in order to effectively prevent any attack.

\section{Experimental Results}

In the performance analysis of the IPS on IPTV, the IPS is installed on an internet television, including the installation of hardware and software. The configuration of the network is set to perform at full capacity. Testing and analysis of the results of system performance are conducted to evaluate the ability of the IDS, as described in the following procedure.

\subsection{The Installation of the Intrusion Prevention System on Internet Protocol Television}

To install the IPS on IPTV, the system has to process the hardware and software installations. The operation of the hardware installation is composed of an analysis of the IPS, the installation of the computer for Linux, the device, and the Linux server, as well as a test of the operation and functionality of the network. Finally, the configuration of the network to be used is set with the system for effectiveness.

\subsubsection{Linux Operation System Installation}

The Feroda (Linux Operation System) is installed for testing of the system.

\subsubsection{Snort Installation}

Snort is network security software that has been continuously developed globally through to its present Version 2.8.0.1. The Snort runs on the Linux Operation System. 


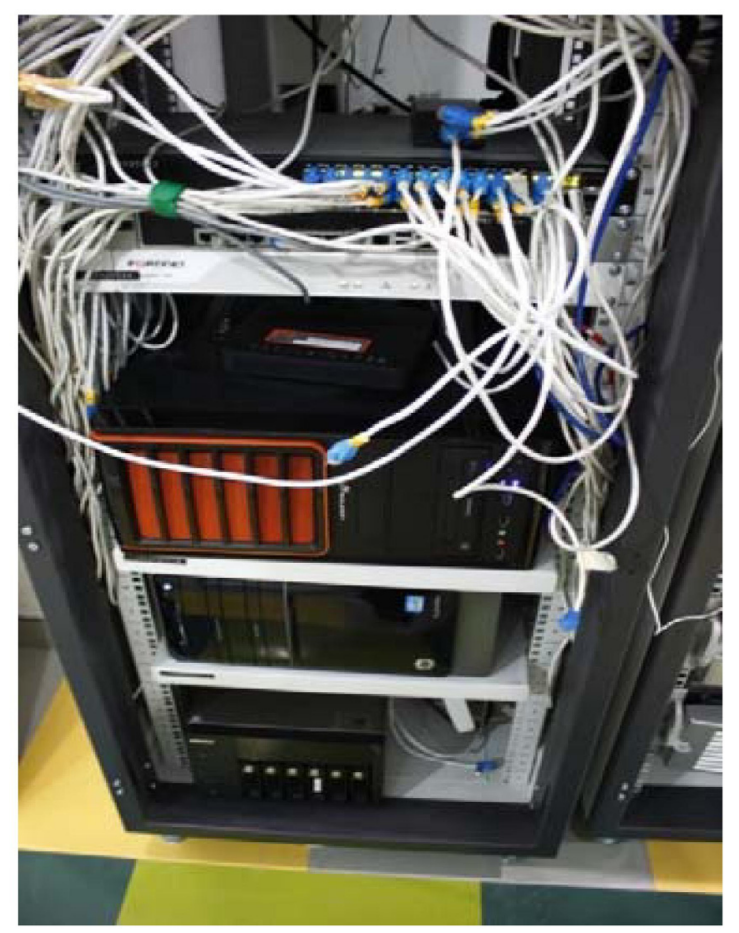

Figure 6. Personal computer installation for Linux server

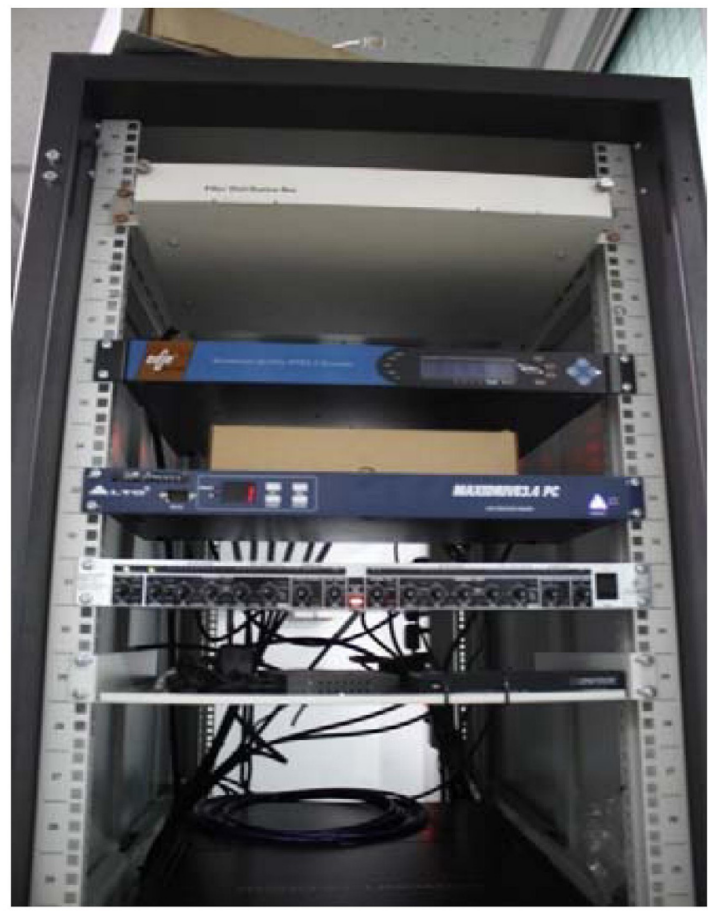

Figure 7. Installation of the network infrastructure

The operation of Snort (Software) installation is started by downloading the Snort program in the version that corresponds with the operating system, then installing Snort via DOS and installing the database for storage Rule Base and its creation. Finally, the web page is installed to show the attack results to the administrator. 


\subsubsection{Snort Database Installation on MySQL}

The Snort stores data into the available database using a variety of software. MySQL was chosen for handling the database because it is open source software and has the PhpMyAdmin program, which makes database management easier. Moreover, it can be developed in the source code.

\subsubsection{Installation of Snort Rule}

The Rule is a regulation used to detect attack patterns or a packet that can be detected by a program. Typically, the program defines the Rule for analysis of simple attack patterns. However, the program does not enable the operation of all Rules. It only uses some Rules that have intensified. It cannot compare the highest performance in detecting attacks on the program. Therefore, the Rule is modified by customizing and configuring the value in Snort.conf file.

\subsection{Comparative Analysis Results}

In the tests of IDS effectiveness, the attack via a wired and wireless network are displayed by six graphs comprised of TCPUnicast IP Address, TCP-Multicasts IP Address, UDPUnicast IP Address, UDP-Multicasts IP Address, ICMPUnicast IP Address, and ICMP-Multicasts IP Address. Each attack uses the number of packets and network attacks equally. The results of the network attacks can be divided as follows:

\subsubsection{TCP-Unicast IP Address}

The network attack was designed by attacking the target machine directly in the form of Unicast IP Address and installing the network system that scans the TCP protocol primarily, as shown in Figure 5.

\begin{tabular}{|l|c|l|l|l|l|l|l|l|l|l|}
\hline TCP Unicast Alerts & \multicolumn{10}{|c|}{ Time } \\
\hline Network & \multicolumn{10}{|c|}{} \\
\hline & $\mathbf{1}$ & $\mathbf{2}$ & $\mathbf{3}$ & $\mathbf{4}$ & $\mathbf{5}$ & $\mathbf{6}$ & $\mathbf{7}$ & $\mathbf{8}$ & $\mathbf{9}$ & $\mathbf{1 0}$ \\
\hline Lan & 35100 & 35600 & 35400 & 36100 & 36200 & 35500 & 36000 & 36700 & 35900 & 36400 \\
\hline Wireless & 35400 & 35700 & 35500 & 35900 & 35700 & 35490 & 35510 & 35520 & 35500 & 35505 \\
\hline
\end{tabular}

Table 1. TCP Unicast Alerts Record

Table 1 records the results of the packet numbers that can trap the attack each time. The table is a record in the form of the TCP Multicast and the wired and wireless network attacks. The attack is tested 10 times to display the graph of experimental results, as shown in Figure 8.

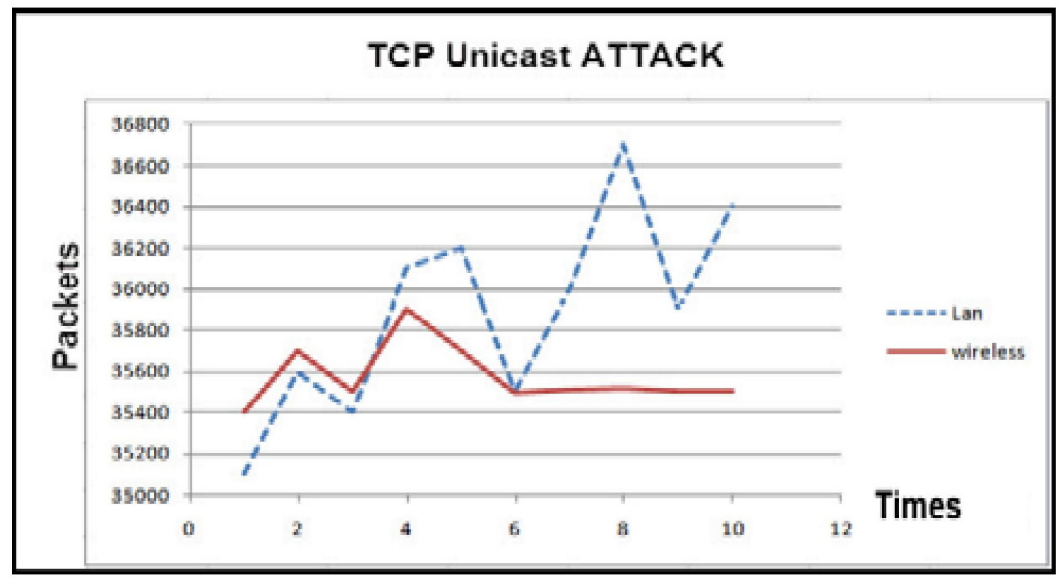

Figure 8. Graph of the TCP Protocol Results with the network attack of Unicast IP Address 
From the graph showing the TCP protocol packet detection from the Unicast IP Address attack, the x-axis is the number of packets that can be detected by the system, while the y-axis is the number of times tested. As shown in the graph, the attack over the wired network can detect the packet with the TCP protocol more than the attack over the wireless network at $0.89 \%$.

\subsubsection{TCP-Multicasts IP Address}

The network attack was designed by attacking the target machine directly in the form of Multicast IP Address and installing the network system that scans the TCP protocol primarily, as shown in Figure 5.

\begin{tabular}{|l|c|l|l|l|l|l|l|l|l|l|}
\hline TCP Multicast Alerts & \multicolumn{7}{|c|}{ Time } \\
\hline Network & \multicolumn{9}{|c|}{} \\
\hline & $\mathbf{1}$ & $\mathbf{2}$ & $\mathbf{3}$ & $\mathbf{4}$ & $\mathbf{5}$ & $\mathbf{6}$ & $\mathbf{7}$ & $\mathbf{8}$ & $\mathbf{9}$ & $\mathbf{1 0}$ \\
\hline Lan & 45000 & 40900 & 43090 & 38100 & 38150 & 38200 & 38100 & 38150 & 38100 & 36100 \\
\hline Wireless & 21100 & 21090 & 21100 & 21100 & 19800 & 14090 & 14090 & 14090 & 14080 & 14070 \\
\hline
\end{tabular}

Table 2. TCP Multicast Alerts Record

From Table 2, the results of the packet numbers that can trap the attack each time are recorded. The table provides a record in the form of the TCP Multicast and the wired and wireless network attacks. The attack is tested 10 times to display the graph of experimental results, as shown in Figure 9.

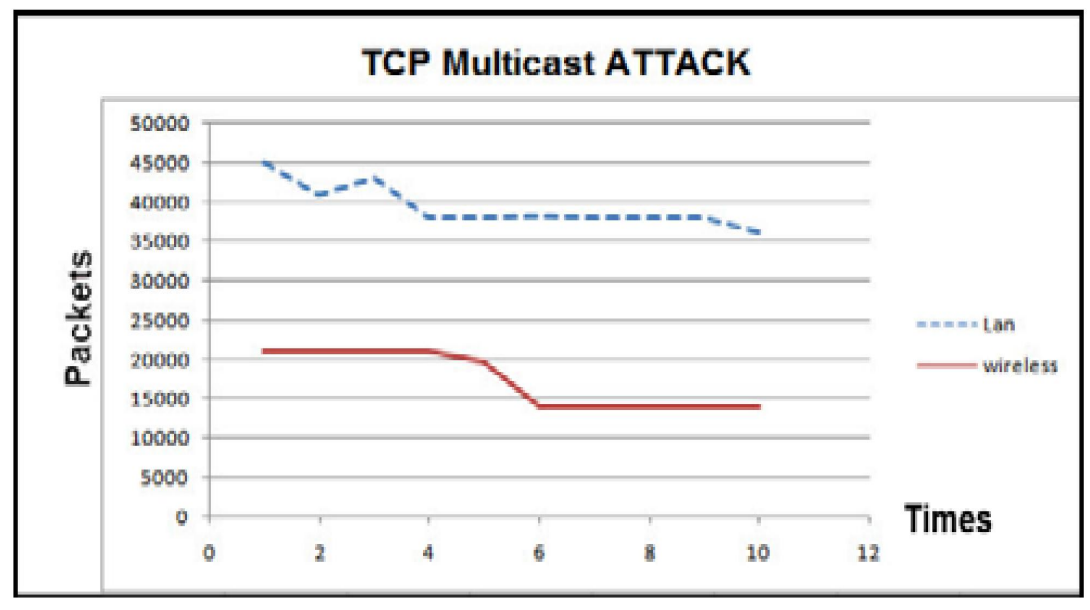

Figure 9. Graph of the TCP Protocol Results with the network attack of Multicast IP Address

From the graph showing the TCP protocol packet detection from the Multicast IP Address attack, the $\mathrm{x}$-axis is the number of packets that can be detected by the system, while the yaxis is the number of times tested. As shown in the graph, the attack over the wired network can detect the packet with the TCP protocol more than the attack over the wireless network at $125.6 \%$.

\subsubsection{UDP-Unicast IP Address}

The network attack was designed by attacking the target machine directly in the form of Unicast IP Address and installing the network system that scans the TCP protocol primarily, as shown in Figure 5.

Table 3 shows recording for the results of the packet numbers that can trap the attack each time. This table is a record in the form of the UDP Unicast and the wired and wireless network attacks. The attack is tested 10 times to display the graph of experimental results, as shown in Figure 10. 


\begin{tabular}{|l|l|l|l|l|l|l|l|l|l|l|}
\hline UDP Unicast Alerts & \multicolumn{9}{|c|}{ Time } \\
\hline Network & \multicolumn{9}{|c|}{} \\
\hline & 1 & $\mathbf{2}$ & $\mathbf{3}$ & $\mathbf{4}$ & $\mathbf{5}$ & $\mathbf{6}$ & $\mathbf{7}$ & $\mathbf{8}$ & $\mathbf{9}$ & $\mathbf{1 0}$ \\
\hline Lan & 190 & 192 & 191 & 195 & 193 & 186 & 180 & 186 & 190 & 199 \\
\hline Wireless & 110 & 105 & 120 & 100 & 90 & 90 & 100 & 89 & 105 & 100 \\
\hline
\end{tabular}

Table 3. UDP Unicast Alerts Record

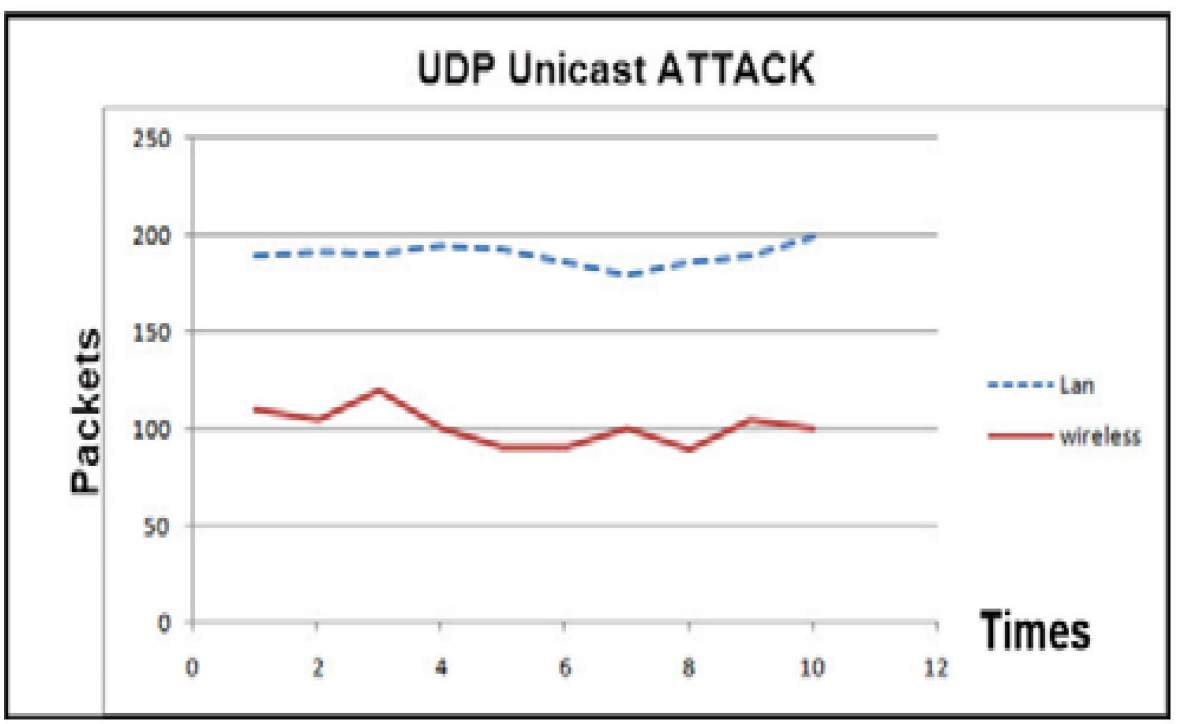

Figure 10. Graph of the UDP Protocol Results with the network attack of Unicast IP Address

From the graph showing the UDP protocol packet detection from the Unicast IP Address attack, the x-axis is the number of packets that can be detected by the system, while the y-axis is the number of times tested. As shown in the graph, the attack over the wired network can detect the packet with the UDP protocol more than the attack over the wireless network at $88.5 \%$.

\subsubsection{UDP-Multicasts IP Address}

The network attack was designed by attacking the target machine directly in the form of Multicast IP Address and installing the network system that scans the UDP protocol primarily, as shown in Figure 5.

\begin{tabular}{|l|l|l|l|l|l|l|l|l|l|l|}
\hline UDP Multicast Alerts & \multicolumn{7}{|c|}{ Time } \\
\hline Network & \multicolumn{9}{|c|}{} \\
\hline & $\mathbf{1}$ & $\mathbf{2}$ & $\mathbf{3}$ & $\mathbf{4}$ & $\mathbf{5}$ & $\mathbf{6}$ & $\mathbf{7}$ & $\mathbf{8}$ & $\mathbf{9}$ & $\mathbf{1 0}$ \\
\hline Lan & 650 & 620 & 619 & 630 & 680 & 640 & 650 & 655 & 640 & 640 \\
\hline Wireless & 199 & 201 & 200 & 210 & 200 & 200 & 205 & 210 & 205 & 199 \\
\hline
\end{tabular}

Table 4. UDP Multicast Alerts Record 
Table 4 shows a record for the results of the packet numbers that can trap the attack each time. This table is a record in the form of the UDP Multicast and the wired and wireless network attacks. The attack is tested 10 times to display the graph of the experimental results, as shown in Figure 11.

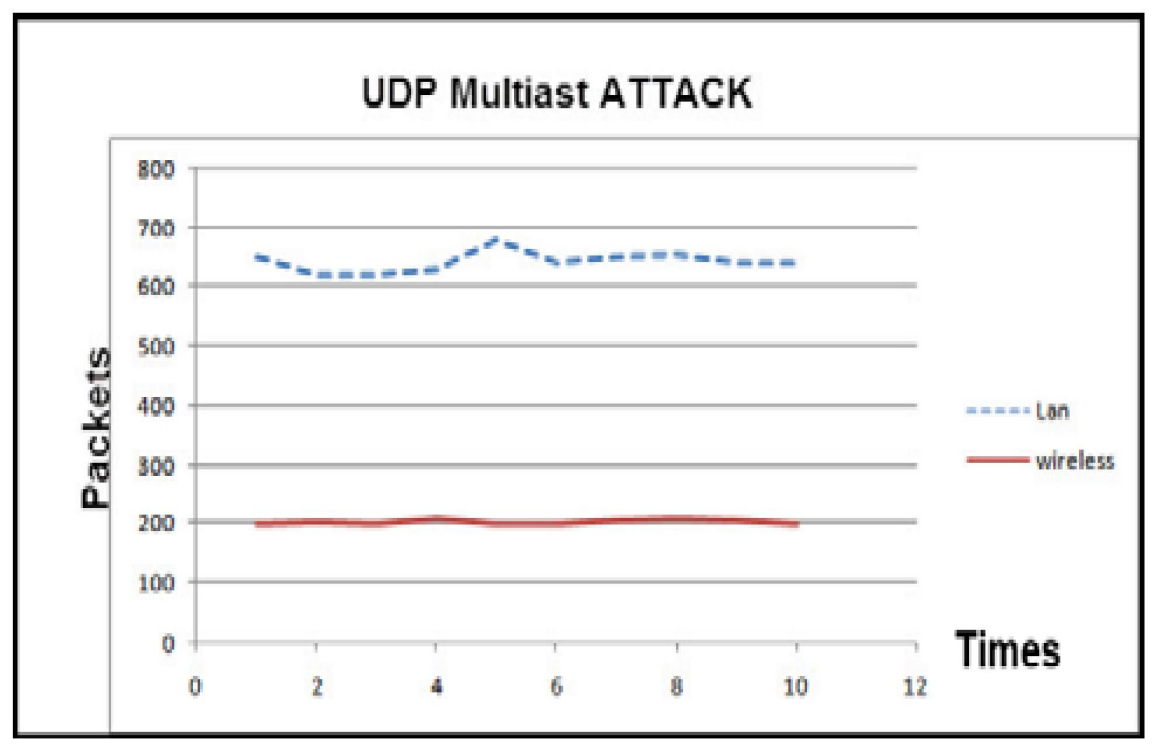

Figure 11. Graph of the UDP Protocol Results with the network attack of Multicast IP Address

From the graph showing the UDP protocol packet detection from the Multicast IP Address attack, the $\mathrm{x}$-axis is the number of packets that can be detected by the system, while the y-axis is the number of times tested. As shown in the graph, the attack over the wired network can detect the packet with the UDP protocol more than the attack over the wireless network at $216.6 \%$.

\subsubsection{ICMP-Unicast IPAddress}

The network attack was designed by attacking the target machine directly in the form of Unicast IP Address and installing the network system that scans the ICMP protocol primarily, as shown in Figure 5.

\begin{tabular}{|l|l|l|l|l|l|l|l|l|l|l|}
\hline ICMP Unicast Alerts & \multicolumn{7}{|c|}{ Time } \\
\hline Network & \multicolumn{10}{|c|}{} \\
\hline & $\mathbf{1}$ & $\mathbf{2}$ & $\mathbf{3}$ & $\mathbf{4}$ & $\mathbf{5}$ & $\mathbf{6}$ & $\mathbf{7}$ & $\mathbf{8}$ & $\mathbf{9}$ & $\mathbf{1 0}$ \\
\hline Lan & 170 & 175 & 175 & 175 & 175 & 175 & 160 & 175 & 175 & 174 \\
\hline Wireless & 60 & 60 & 60 & 55 & 55 & 55 & 55 & 60 & 55 & 55 \\
\hline
\end{tabular}

Table 5. ICMP Unicast Alerts Record

Table 5 shows a record for the results of the packet numbers that can trap the attack each time. This table is a record in the form of the ICMP Unicast and the wired and wireless network attacks. The attack is tested 10 times to display the graph of the experimental results, as shown in Figure 12.

From the graph showing the ICMP protocol packet detection from the Unicast IP Address attack, the x-axis is the number of packets that can be detected by the system, while the yaxis is the number of times tested. As shown in the graph, the attack over the wired network can detect the packet with the ICMP protocol more than the attack over the wireless network at $203.3 \%$. 


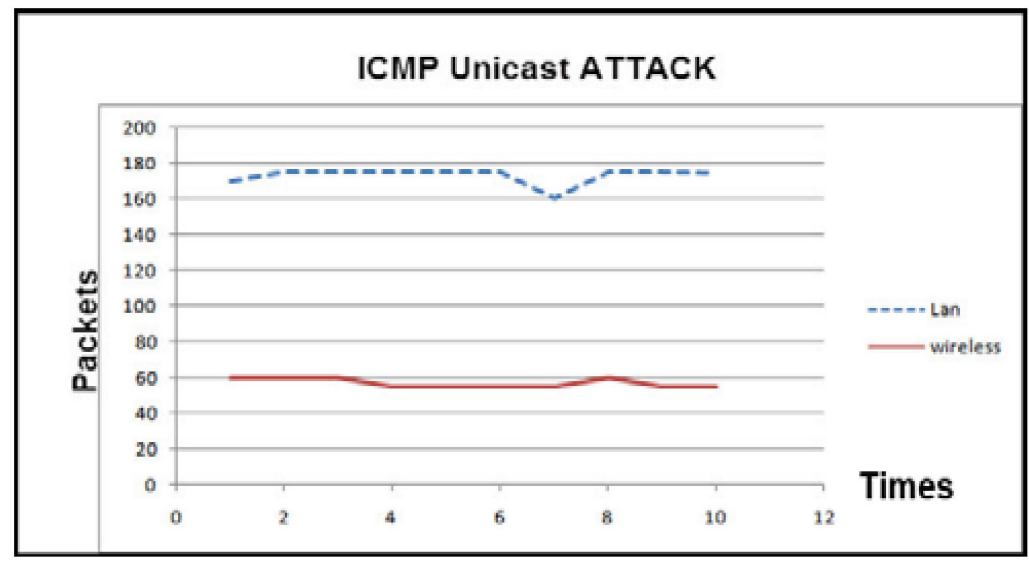

Figure 12. Graph of the ICMP Protocol Results with the network attack of Unicast IP Address

\subsubsection{ICMP-Multicasts IP Address}

The network attack was designed by attacking the target machine directly in the form of Multicast IP Address and installing the network system that scans the ICMP protocol primarily, as shown in Figure 5.

\begin{tabular}{|l|c|l|l|l|l|l|l|l|l|l|}
\hline ICMP Multicast Alerts & \multicolumn{7}{|c|}{ Time } \\
\hline Network & \multicolumn{9}{|c|}{} \\
\hline & $\mathbf{1}$ & $\mathbf{2}$ & $\mathbf{3}$ & $\mathbf{4}$ & $\mathbf{5}$ & $\mathbf{6}$ & $\mathbf{7}$ & $\mathbf{8}$ & $\mathbf{9}$ & $\mathbf{1 0}$ \\
\hline Lan & 690 & 600 & 480 & 580 & 575 & 599 & 586 & 610 & 595 & 594 \\
\hline Wireless & 185 & 200 & 185 & 199 & 200 & 199 & 200 & 200 & 199 & 199 \\
\hline
\end{tabular}

Table 6. ICMP Multicast Alerts Record

Table 6 is a record for the results of the packet numbers that can trap the attack each time. The table is a record in the form of the ICMP Multicast and the wired and wireless network attacks. The attack is tested 10 times to display the graph of the experimental results, as shown in Figure 13.

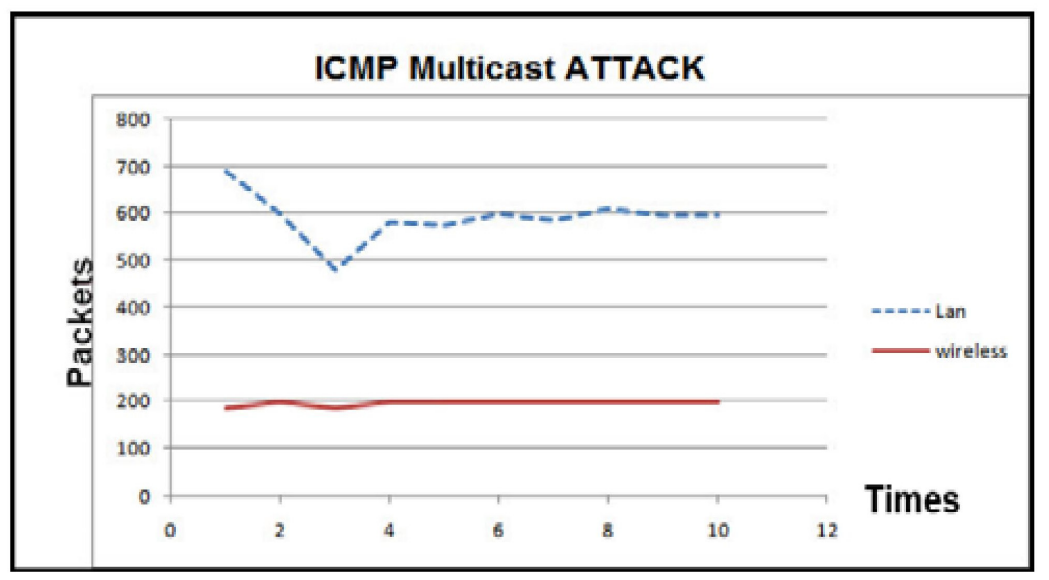

Figure 13. Graph of the ICMP Protocol Results with the network attack of Multicast IP Address 
From the graph showing the ICMP protocol packet detection from the Multicast IP Address attack, the x-axis is the number of packets that can be detected by the system, while the y-axis is the number of times tested. As shown in the graph, the attack over the wired network can detect the packet with the ICMP protocol more than the attack over the wireless network at $200.5 \%$.

\section{Conclusions}

The results for the analysis of the network IPS on IPTV with the intrusion prevention techniques in the part of internal attack are shown below:

\begin{tabular}{|l|l|l|l|}
\hline Network & TCP & UDP & ICMP \\
\hline Wireless (unicast) & 35572.5 & 100.9 & 57 \\
\hline Wireless (Multicast) & 17461 & 202.9 & 196.6 \\
\hline LAN (unicast) & 35890 & 190.2 & 172.9 \\
\hline LAN (Multicast) & 39389 & 642.4 & 590.9 \\
\hline
\end{tabular}

Table 7. A Summary of Average Attacks

From Table 7, it could be concluded that the TCP protocol is able to detect and prevent possible attacks because an attack for Denial of Service has more vulnerability than UDP and ICMP protocols. When considering the attack patterns, the attack via wired network is more easily detected than via the wireless network because of the access point. The wireless network has an access point used to detect information retrieval. Thus, the packet will filter information before accessing it. The Multicast network has more efficiency than the Unicast network. The Multicast is used for a variety of attacks over the network unlike the Unicast, which has a specific network attack. Therefore, Unicast is not a channel of attack by intruders.

From the experimental results, it was found that the network IPS on IPTV with the application of Snort was the most effective for preventing attacks from intruders when using the TCP protocol. The TCP attacks the wired network with Multicast IP Address, as shown in Table 7. It can detect the packets of attack by up to 39,389 packets, meaning that the use of the IPS helps reduce and prevent attacks. However, the IPS should be placed in other positions and compared to identify the best performance. In the experimental study and actual implementation, the experiment in the internal system has a limited time. Thus, the test was done 10 times within the time limit. As a result, no difference between limited and fulltime testing was found.

\section{References}

[1] Association of Southeast Asian Nations. ASEAN Ministers Responsible for Information (AMRI), http://asean.org/ ?static_post=joint-media-statementeleventh-conference-of-the-asean-ministers-responsiblefor-information-11th-amri-andsecond-conference-ofasean-plus-three-ministers-responsible-for-information-2nd-amri3-kuala-1

[2] Point Topic Ltd, http://point-topic.com/free-analysis/iptvsubscribers-market-analysis-q4-2015/

[3] Jabbar, H., Jeong, T.-Y., Hwang, J., Park, G.-L. (2008). Viewer identification and authentication in IPTV using RFID technique, IEEE Transactions on Consumer and Electronics, 54 (1) 105-109.

[4] Jun, E.-A., Kim, J. G., Jung, S. W., Lee, D. H. (2011). Extended fingerprint-based user authentication scheme using smart cards in education IPTV, In: Proceedings of the International Conference on Information Science and Applications (ICISA '11), p. 17, Jeju Island, Republic of Korea, (April).

[5] Yoo, H. J. (2012). Secure mutual authentication for IPTV broadcasting, In: Proceedings of the International Conference on ICT Convergence (ICTC'12), p. 96-99, IEEE, (October).

[6] Tibin, L. (2010). Analyzes IPTV Security Threats under the Triple Play. In: Proceedings of the International Conference on Multimedia Information Networking and Security (MINES'10), p. 720-724. IEEE, (November). 
[7] Cho, D-E., Yeo, S. S. (2011). IPTV and Secure Digital Contents Downloading. In: Proceedings of the 14th International Conference on Network-Based Information Systems (NBiS'11), p. 582-585. IEEE, (September).

[8] Chung, M., Lee, Y., Shon, T., Moon, J. (2013). A security model for IPTV with one-time password and Conditional Access System for smart mobile platform. Journal of Telecommunication Systems 52 (2) 1399-1407.

[9] Doh, I., Chae, K-J., Lim, J., Chung, M. Y. (2012). An Improved Security Approach Based on Kerberos for M2M Open IPTV System. In: Proceedings of the 15th International Conference on Network-Based Information Systems (NBiS'12), p. 754-759. IEEE, (September).

[10] Pomsathit, A. (2013). Performance Measurement of Intrusion Detection System with a Honeypot over Networking. In: Proc. of the National Conference on Computer Information Technologies (CIT’13), p. 44- 47.

[11] Pomsathit, A. (2012). Performance Evaluation of DoS Attack on Wire and Wireless Network Over Intrusion Detection System with a Honeypot. In: Proceedings of the International Conference on Electrical, Computer, Electronics and Communication Engineering (ICECECE'12), World Academy of Science, Engineering and Technology, (November). 